\title{
Prognostic Analysis and Risk Factors Associated with Fetal Ventriculomegaly
}

\author{
Qi-Ming Li ${ }^{a}$,b Hong-Zhan Liao ${ }^{b}$ Wen-Bo Wang ${ }^{b}$ Shi-Yi Zeng ${ }^{b}$ \\ Xian-Sheng Qiu ${ }^{b}$ Shuai Ke ${ }^{b}$ Jing Xiao ${ }^{b}$ Qing-Hua Lic $^{c}$ Xue-Wei Xiab \\ Yong Lib, d
}

${ }^{a}$ Department of Neurosurgery, Taihe Hospital, Shiyan, China; ${ }^{b}$ Department of Neurosurgery, Affiliated Hospital of Guilin Medical University, Guilin, China; ' Department of Neurology, Affiliated Hospital of Guilin Medical University, Guilin, China; ${ }^{\mathrm{d} S p o r t}$ and Health College of Guangxi Normal University, Guilin, China

\section{Keywords}

Fetal ventriculomegaly · Prenatal diagnosis · Pregnancy outcomes · Risk factor

\begin{abstract}
Background: This study aimed to investigate the clinical outcome and related risk factors of fetal lateral ventriculomegaly (VM). Methods: A retrospective analysis was performed on 255 cases diagnosed as fetal VM. Prenatal imaging examination was carried out. The pregnancy outcomes were investigated through follow-up. According to the prognosis of children, they were divided into case group and control group. Multivariate logistic regression was used to analyze the factors influencing the prognosis of hydrocephalus. Results: After excluding the cases with either loss of follow-up or incomplete information, 102 cases were followed up. Twelve cases with poor prognosis were set as the case group. According to the maternal age, gestational age, gender of children, and follow-up time, 3 cases were selected from the other 90 cases for each child in the case group, respectively, and selected as the control group. Paired comparative analysis was performed on 48 cases. Using prognosis as a dependent variable, multivariate logistic regression
\end{abstract}

analysis of the statistically significant factors indicated that the change speed of width ratio (CSWR) and maximum lateral ventricular width (MW) were associated with fetal prognosis. Conclusions: Our results suggested that CSWR and MW may have the value of predicting fetal prognosis.

(c) 2021 S. Karger AG, Base

\section{Background}

Ventriculomegaly (VM) is considered as the most common malformation in the fetal central nervous system development, with an incidence rate of 7.03/10,000. Based on the degree of lateral ventricular dilatation, it is usually classified into mild $(10 \sim 12 \mathrm{~mm})$, moderate $(13 \sim 15 \mathrm{~mm})$, and severe (>15 $\mathrm{mm})$ [1].

Severe fetal lateral ventricular dilatation has impact on the survival of children after birth and may lead to maternal dystocia. Partial VM has been discovered to be frequently associated with the primary central nervous system developmental disorders, which are inclusive of my-

Qi-Ming Li and Hong-Zhan Liao contributed equally to this work. 
elomeningocele, arachnoid cyst, etc. [2]. The simple VM with no associated anomaly is known as an isolated VM (IVM). Following the study of 167 cases of fetal isolated mild VM, Ouahba et al. [3] drew a conclusion that the VM prognosis was associated with various factors, including atrial width $>12 \mathrm{~mm}$, progression of enlargement, as well as asymmetrical and bilateral VM [3].

However, Bar-Yosef [4] believed that the IVM neurodevelopmental outcome was generally better, and there was no correlation between the prognosis and the severity or asymmetry of VM. A majority of scholars take the belief that mild IVM will develop into asymptomatic hydrocephalus after the birth of most infants, and the prognosis of fetus with large ventricle width or other complications remains debatable [3-5]. Therefore, early diagnosis and early intervention are deemed critical for fetal VM. In this study, a retrospective study of 255 cases with fetal VM was performed to examine the prognosis and intrauterine outcome of VM, and to analyze its risk factors, with an aim at providing an effective basis for clinical diagnosis and following treatment.

\section{Methods}

Design

In the study, a randomized controlled trial was conducted to ascertain the relationship between ventricle width and its changes in fetus as well as the survival status of the infant after birth. A hypothesis was made that excessive or rapid changes in fetal lateral ventricle width may be a risk factor that could affect the prognosis of infants after delivery.

\section{Participants}

This randomized controlled clinical trial was approved by the Institutional Review Board of Affiliated Hospital of Guilin Medical University (Guilin, China) and in accordance with the World Medical Association Declaration of Helsinki. Written informed consent was obtained from all patients' parents. From January 2014 to January 2017, a total of 164 participants were recruited in the outpatient department of obstetrics at the Affiliated Hospital of Guilin Medical College, which was rated as a large comprehensive hospital where in excess of five thousand pregnant women were tested annually. Inclusion criteria: (1) color Doppler ultrasound or magnetic resonance imaging (MRI) confirmed lateral ventricular dilatation $>10 \mathrm{~mm}$; (2) the gestational age was between 15 and 33 weeks; (3) single pregnancy; and (4) monthly color doppler ultrasound examination ( 3 months at least). Exclusion criteria: (1) incomplete imaging data or unclear diagnosis; (2) the external factors influencing the prognosis of children included birth injury, hypoxic-ischemic encephalopathy, and intrauterine asphyxia, etc.; (3) identifying other diseases that affect the prognosis (e.g., intraventricular hemorrhage and posthemorrhagic hydrocephalus); (4) preterm birth (gestational age $<33$ weeks at birth); and (5) chromosomal abnormality or TORCH infection.
Table 1. Clinical characteristics of 164 fetal cases with VM

\begin{tabular}{lr}
\hline Clinical characteristics & $N(\%)$ \\
\hline Maternal age, yr & \\
$\quad<35$ & $142(86.6)$ \\
$\quad \geq 35$ & $22(13.4)$ \\
Gestational age at diagnosis, wk & \\
$\quad<24$ & $46(28.0)$ \\
$\quad \geq 24$ & $118(72.0)$ \\
Lateral ventricle width, mm & \\
$\quad<12$ & $125(76.2)$ \\
$\quad \geq 12$ & $39(23.8)$ \\
Lateral ventricle & $116(70.7)$ \\
Unilateral & $48(29.3)$ \\
Bilateral & \\
Intrauterine change & $62(37.8)$ \\
TOP & $32(19.5)$ \\
Recovery & $6(3.7)$ \\
Decreased & $24(14.6)$ \\
Progressive & $40(24.4)$ \\
Stable & \\
\hline VM, ventriculomegaly. & \\
\hline
\end{tabular}

\section{Examination Method}

The examination was carried out by using GE-Volusion E10 color Doppler ultrasound diagnostic instrument. Ultrasound screening of fetal malformations was conducted as a routine for pregnant women who were enrolled in our hospital during the gestational week. The VM can be diagnosed using the color Doppler ultrasonography or MRI when the lateral ventricle width $>10$ $\mathrm{mm}$. Lateral ventricle width is the widest distance. The atrium of the lateral ventricle should be measured in the transventricular (axial) plane at the level demonstrating the frontal horns and cavum septi pellucidi, in which the cerebral hemispheres are symmetric in appearance. The calipers should be positioned on the internal margin of the medial and lateral walls of the atria, at the level of the parietal-occipital groove and glomus of the choroid plexus, on an axis perpendicular to the long axis of the lateral ventricle. The color Doppler ultrasonography was performed by 2 experienced doctors in the color Doppler ultrasonic room of the hospital. It is required that the pregnant woman undergo an MRI examination when suspected congenital malformations of the central nervous system are discovered. The MRI examination was performed by 2 experienced doctors in the imaging department of the hospital. The fetuses with VM were reexamined by using ultrasonography every 2-4 weeks (at least 3 times, except for those who induced labor). The changes to the width of lateral ventricles were measured to make assessment of the intrauterine outcome of VM until birth. And the infant should be reexamined by using the MRI examination at 1 week and 1 month after birth.

Progressive analysis of fetal imaging data classifies the fetus as progressive when the fetal lateral ventricular width increases $>5$ mm during pregnancy, or when the fetal width of lateral ventricular increases by $3 \mathrm{~mm}$ within a month. The morphology of fetal lateral ventricles (unilateral or bilateral) was recorded. The results of color ultrasound and MRI results of pregnant women were re- 
Table 2. Twelve children with poor prognosis

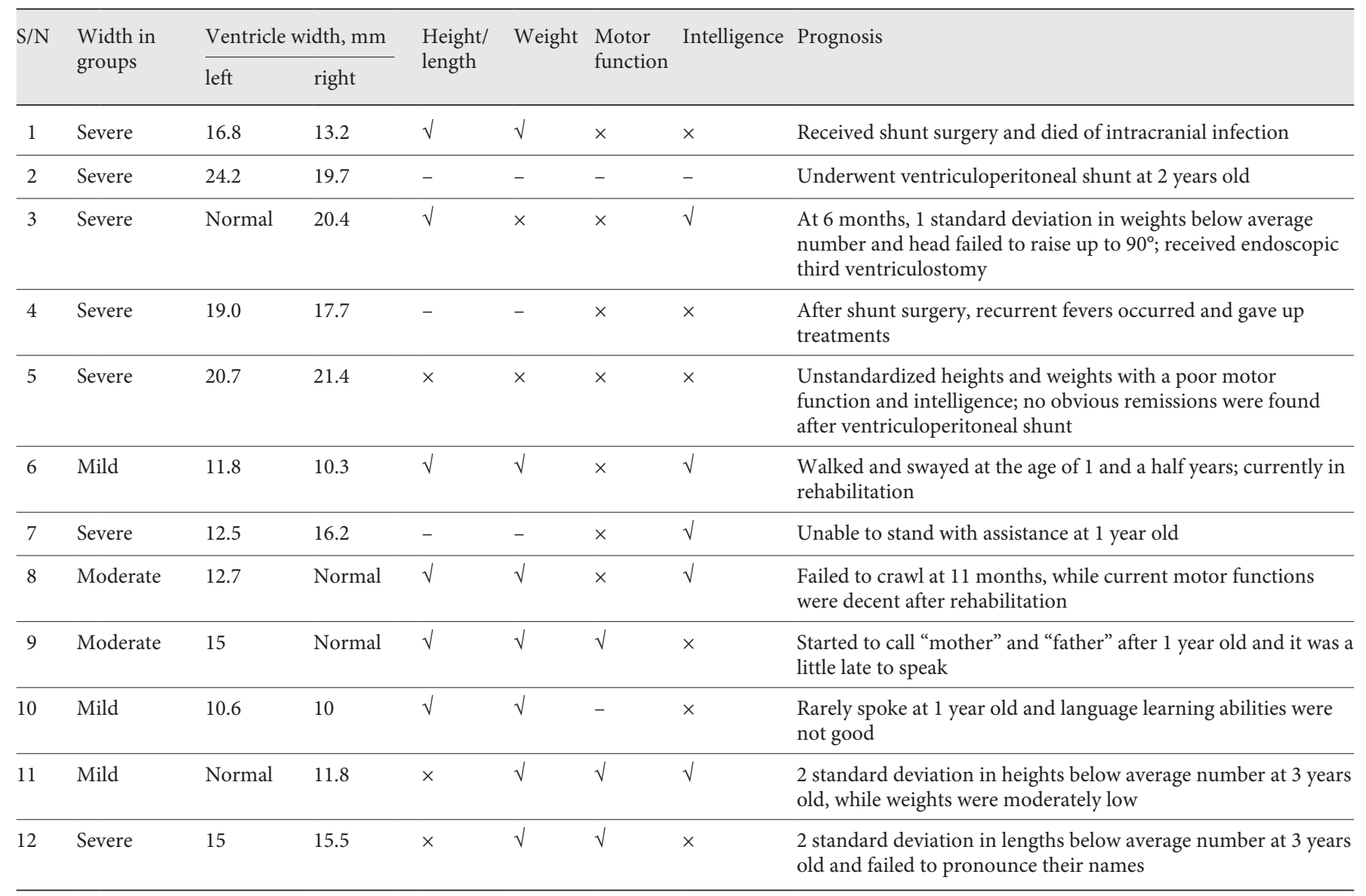

$\sqrt{ }$ means qualified; $\times$ means unqualified; - means not done.

viewed to ascertain whether they were combined with other neurological malformations, such as arachnoid cyst, congenital spina bifida, or congenital absence of corpus callosum. Depending on the maximum width of lateral ventricle, the fetus was categorized into mild (>10 and $\leq 12 \mathrm{~mm}$ ), moderate, and severe ventricular dilatation ( $>12 \mathrm{~mm}$ ) because many scholars believe that the fetus may have the worse prognosis when the lateral ventricle separation $>12 \mathrm{~mm}$.

The relevant information about the pregnant women was recorded, including the maternal age, gestational age at diagnosis, gender of fetus, and follow-up time. The variables associated with fetal lateral ventricle were measured, including degree of expansion, progression, ventricle (unilateral or bilateral), presence or absence of other nervous system malformations, maximum lateral ventricular width (MW), width difference of VM, and change month.

\section{Follow-Up}

The follow-up doctor, a neurosurgeon, conducted a telephone follow-up and analyzed the results obtained from the postnatal reexamination. The growth and development of the children were assessed based on the WHO Standards of Child Growth. The mo- tor and language functions of the children were assessed according to the Denver developmental screening test. In case that any of the growth and development indicators, motor function assessment and language function assessment failed to meet the normal criteria, the child was considered as symptomatic infant hydrocephalus, which was then classified into the case group. If the child had a relevant record of surgery (ventriculoperitoneal shunt or neuroendoscopic third ventriculostomy), he or she was also classified into the case group. When the growth and development indicators, motor function assessment, and language function assessment were found to be normal, the prognosis of the child was considered as good and classified into the control group.

\section{Statistical Analysis}

Statistical analysis was conducted using SPSS 22.0 software (International Business Machines, Corp., Armonk, NY, USA). The measured data conforming to the normal distribution were expressed as $\pm \mathrm{s}$, and the $t$ test was carried out for comparison between the groups. The counting of data was expressed as frequency and percentage. The $\chi^{2}$ test or Fisher's exact test was performed for comparison between the groups. The odds ratio and $95 \%$ confidence interval were involved to assess the correlation between 
Table 3. Personal information of 48 cases of fetal VM

\begin{tabular}{lccc}
\hline Clinical features & Case group & Control group & $p$ value \\
\hline Maternal age, year & $29.8 \pm 4.4$ & $29.1 \pm 4.9$ & 0.570 \\
Gestational age at diagnosis, week & $27.2 \pm 4.4$ & $27.1 \pm 4.8$ & 0.591 \\
Follow-up time, year & $2.2 \pm 0.7$ & $2.7 \pm 0.7$ & 0.880 \\
Gender (male/female) & $7 / 5$ & $7 / 5$ & 0.721 \\
\hline
\end{tabular}

VM, ventriculomegaly.

Table 4. Clinical features of 48 cases of fetal VM (dichotomous variables)

\begin{tabular}{|c|c|c|c|c|c|}
\hline Factors & Category & $\begin{array}{l}\text { Case } \\
\text { group, } n\end{array}$ & $\begin{array}{l}\text { Control } \\
\text { group, } n\end{array}$ & $\chi^{2}$ & $p$ value \\
\hline Lateral ventricles & $\begin{array}{l}\text { Unilateral } \\
\text { Bilateral }\end{array}$ & $\begin{array}{l}4 \\
8\end{array}$ & $\begin{array}{l}23 \\
13\end{array}$ & 3.343 & 0.723 \\
\hline Degree of expansion & $\begin{array}{l}\leq 12 \\
>12\end{array}$ & $\begin{array}{r}2 \\
10\end{array}$ & $\begin{array}{r}28 \\
8\end{array}$ & 8.874 & $0.025^{*}$ \\
\hline Combined with other neurological malformations & $\begin{array}{l}\text { Presence } \\
\text { Absence }\end{array}$ & $\begin{array}{r}2 \\
10\end{array}$ & $\begin{array}{r}3 \\
33\end{array}$ & 0.656 & 0.125 \\
\hline Progressive VM & $\begin{array}{l}\text { Presence } \\
\text { Absence }\end{array}$ & $\begin{array}{l}6 \\
6\end{array}$ & $\begin{array}{r}4 \\
32\end{array}$ & 8.081 & $0.000^{*}$ \\
\hline
\end{tabular}

VM, ventriculomegaly. * Indicates statistical difference.

the influencing factors and the prognosis of lateral ventricle dilatation. $p<0.05$ suggests that the difference is of statistical significance. The variables with statistical variances were analyzed by binary multivariate logistic regression. With prognosis as dependent variable and progress VM, degree of expansion, width difference of lateral ventricle, MW, width ratio, change speed of width difference and change speed of width ratio (CSWR) as independent variables, and multivariate logistic regression analysis was conducted.

\section{Results}

After excluding cases of missing visits or incomplete information, a total of 164 pregnant women were collected. The age of pregnant women was $(28.4 \pm 4.9)$ years, of which 22 women $(13.4 \%)$ were aged over 35 . For the first time, the mean gestational weeks of fetal VM were $(25.7$ \pm 3.7) weeks. As shown in Table 1, 62 cases (62/164, $37.8 \%$ ) chose induced labor, 32 cases (32/164, 19.5\%) observed wide lateral ventricle disappeared in the intrauterine period, 6 cases $(6 / 164,3.7 \%)$ fetal VM declined as compared to the past, 24 cases $(24 / 164,14.6 \%)$ lateral ventricle width showed progression, and the other 40 cases $(40 / 164,24.4 \%)$ child lateral ventricle width remained stable in the prenatal period.

The fetus was followed up for 1-4 years after birth, excluding cases of induced labor, 102 cases were actually followed up. There were 12 cases with clinical symptoms, and 90 cases with no symptoms exhibited. As shown in Table 2, out of 102 cases, 5 cases (4.9\%) were treated by surgery for different reasons, 3 cases $(2.9 \%)$ with motor dysfunction, one of them was improved after rehabilitation treatment, 2 cases (2\%) with language learning difficulties or slower time to speak than their peers, 1 case $(1.0 \%)$ with slower growth and development than their peers, and 1 case $(1.0 \%)$ with slower growth and poorer language skills than their peers.

Twelve children with poor prognosis were classified into the case group, 90 children with good prognosis were classified into the control group, and 3 controls were selected for each child in the case group according to the maternal age, gestational age, child gender, and follow-up 
Table 5. Clinical features of 48 cases of fetal VM (continuous variables)

\begin{tabular}{lrrr}
\hline Factors & Case group & Control group $p$ value \\
\hline MW, mm & $16.8 \pm 4.2$ & $11.9 \pm 2.3$ & $0.013^{*}$ \\
Width difference of lateral ventricle (WD), mm & $5.3 \pm 2.5$ & $0.5 \pm 3.5$ & $0.000^{*}$ \\
Width ratio (WR) (|WD/MW|) & $0.5 \pm 0.6$ & $0.2 \pm 0.1$ & $0.010^{*}$ \\
Change month (CM), month & $2.3 \pm 0.7$ & $2.5 \pm 0.9$ & $>0.05$ \\
Change speed of width difference (CWD) (WD/CM), mm/month & $2.3 \pm 0.7$ & $0.1 \pm 1.4$ & $0.001^{*}$ \\
CSWR (WR/CM), /month\% & $1.4 \pm 5.6$ & $9.1 \pm 1.2$ & $0.007^{*}$ \\
\hline
\end{tabular}

CSWR, change speed of width ratio; MW, maximum lateral ventricular width; VM, ventriculomegaly. * Indicates statistical difference.

Table 6. Result of multivariate logistic regression analysis

\begin{tabular}{llcc}
\hline Factor & OR & 95\% CI & $p$ value \\
\hline MW & 1.451 & $1.100 \sim 1.915$ & 0.008 \\
CSWR & 1.244 & $1.039 \sim 1.489$ & 0.017 \\
Progressive VM & 5.401 & $0.299 \sim 97.417$ & 0.253 \\
Degree of expansion & 0.208 & $0.006 \sim 7.439$ & 0.389 \\
Width ratio & 4.700 & $0.020 \sim 1,110.846$ & 0.579 \\
Width difference of lateral ventricle & 0.934 & $0.135 \sim 6.468$ & 0.945 \\
Change speed of width difference & 0.895 & $0 \sim 74,672.914$ & 0.985 \\
\hline
\end{tabular}

CSWR, change speed of width ratio; MW, maximum lateral ventricular width; VM, ventriculomegaly; CI, confidence interval.

time. A paired comparative analysis was performed on these 48 children (Table 3 ).

The dichotomous variables were tested by the $\chi^{2}$ test. After verifying that the continuous variables conformed to the normal distribution, the $t$ test was carried out (Tables 4 , 5). The variables with statistical variances were analyzed by binary multivariate logistic regression, and the results revealed that the CSWR and MW bore relation with fetal prognosis, while no other variables were discovered to be associated with prognosis in this study (Table 6).

\section{Discussion}

Congenital fetal VM is regarded as a common congenital central nervous system lesion in neonates. The treatment of symptomatic congenital VM among children has always been a challenge for neurosurgery, which not only leads to developmental disorder for children, but also increases social and family burdens. Therefore, at- tention is worth being paid to prenatal screening for such congenital diseases in order to reduce their incidence, and provide effective guidance and intervention for pregnant women in prenatal care.

Ultrastructural changes in the brain tissue resulting from fetal VM are manifested as the destruction of the ependymal lining on the surface of the ventricular wall [6]. Cohesive multi-ciliated epithelium that is formed by ependymal cells epithelium acts as a bidirectional barrier between the ventricle and the brain parenchyma and transports CSF components and interstitial fluids between these domains [6, 7]. Tight cell-to-cell adhesion between ependymal cells is mediated by cadherin-based adherens junctions situated at the subapical membrane, which is essential for ventricular integrity $[8,9]$. Many studies suggest that ependymal cell denudation is emerging as a major cause of hydrocephalus [7,9]. Therefore, promoting the adhesion of ependymal cells could be the way to improve hydrocephalus. O'Leary et al. [10] indicate that adherens junction assembly relies on neogenin 
recruitment of the WRC and Arp2/3 to promote actin polymerization and that its loss results in hydrocephalus. Neogenin therefore stabilizes ependymal junctions to buffer the forces generated by increasing the CSF in the neonate [10]. Szabolcsi and Celio [11] show that the injury-triggered upregulation of PV expression is mediated by inflammatory cytokines, and promotes the motility and adhesion of ependymal cells, thereby contributing to leakage closure by the reestablishment of a continuous ependymal layer. VM is reversible for brain tissue damage during the initial phase. Throughout this period, the damaged brain tissue is capable of restoring its function when the width of lateral ventricle decreases. However, the further expansion will make such a damage irreversible, which is known as the "threshold effect" [12]. When the ventricle reaches a certain threshold, the ependymal lining fiber cannot continue expansion, which will result in a long-term damage. The severity of this permanent injury is contributed by a variety of different factors, including the gestational age, duration of illness, degree of ventricular dilatation, ventricular size, intracranial pressure, and other neurological lesions in the brain. Between 15 and 40 weeks of gestation, the width of the lateral ventricle is about $5.4-7.6 \mathrm{~mm}$ on average, so when the ventricle width is equated to $10 \mathrm{~mm}$, it signifies that the value is $2.5 \sim 4$ times the standard deviation of the mean, which often indicates a kind of abnormal development of the nervous system [13]. The standard plane for measuring the width of the lateral ventricle is positioned transversely, the bilateral cerebral hemispheres are symmetrical, and the anterior horn of the lateral ventricle and the cavity of septum pellucidum can be viewed. The distance between the inner wall and the outer wall of the lateral ventricle was measured perpendicular to the long axis of the lateral ventricle. An appropriate method of measurement is required; otherwise, it may give false positive or false negative results. For pregnant women with fetal VM, it is advisable to conduct further MRI examination, which could assist with clinical decision-making [14].

In this study, as indicated by the multivariate logistic regression analysis, the maximum width of the lateral ventricle in the fetal period was one of the risk factors in poor prognosis after birth. In 102 cases of fetal VM, the prognosis of the lateral ventricle with a width $>12 \mathrm{~mm}$ was statistically significant as compared to that of the fetal lateral ventricle with a width $<12 \mathrm{~mm}$, and the prognosis of the latter was noticeably better than that of the former. It is generally understood that mild to moderate IVM has a better prognosis if the lateral ventricular width has no apparent progression in the later stage of the disease [4]. A long follow-up study led some scholars to discover that the prognosis of mild IVM fetuses was better than that of the moderate IVM fetuses [15]. The survival rate of mild IVM neonates ranged between 93 and 98\%, and the proportion of normal nervous system development exceeded $90 \%$. The survival rate of moderate IVM neonates ranged from 80 to $97 \%$, and the proportion of normal nervous system development reached between 75 and $93 \%$. Therefore, there is a notable variation between the prognosis of mild IVM and moderate IVM, and the prognosis of mild IVM is normal in most cases. Therefore, some people take the belief that if there are no other structural abnormalities and the width of the lateral ventricle is within the range of $10-12 \mathrm{~mm}$, excluding genetic diagnostic tests and chromosome screening abnormalities, it is possible to be a normal physiological variation, which is because a majority of them will absorb and improve by themselves during pregnancy. Falip et al. [16] performed a prospective study to analyze the prognosis of 101 IVM fetuses. The prognosis of the mild group was significantly better than that of the moderate group, which was similar to the results obtained from this study [16]. However, the effect of mild to moderate VM on prognosis remains controversial. Chu et al. [17] followed up 241 cases of IVM, and made a discovery that the percentage of poor prognosis in mild, moderate, and severe groups was $11.4,6.7$, and $40 \%$, respectively. The prognosis of the mild group was better than that of the moderate group. It is generally believed that VM will influence the prognosis of the fetus, where the lateral ventricle dilation $>15 \mathrm{~mm}$ has a poor prognosis, and induced labor is generally advocated. Carta et al. [18] performed a meta-analysis of 11 studies, which led to a discovery of 110 cases with severe fetal expansion of the lateral ventricle, including 15 cases of reported stillbirth or neonatal death, 17 cases of mild/moderate disability, and 37 cases of severe disability. Besides, $63 \%$ of the 110 cases of severe fetal VM had poor prognosis, indicating that the mortality and disability rates of severe fetal VM are high. As some pregnant women may choose to terminate their pregnancies during prenatal screening, the actual mortality and disability rate is likely to be higher than reported.

As estimated by some scholars, if the lateral ventricular width progresses in the course of disease, the cases that worsen after birth account for approximately $16 \%$ [19]. If the dilated lateral ventricle remains stable or shrinks or even disappears during pregnancy, the prognosis of such children is normally better [20]. Although 
mild to moderate VM generally does not require surgical treatment after birth, if the lateral ventricular width further progresses and deteriorates, a need may arise for the newborn to have surgical intervention after birth. Chu et al. [17] was prospective in researching 148 cases with IVM ranging from 10 to $15 \mathrm{~mm}$, which led to the discovery that after the birth of fetuses with progressive lateral ventricular width, the number of infants with symptoms of neurobehavioral development increased significantly as compared to that with disappeared, decreased, or stabilized width. Therefore, she was convinced that progressive VM indicated poor prognosis of fetuses. The author followed up 102 cases of fetal VM, finding that 19 cases $(19 / 102,18.6 \%)$ had progressive VM, and 6 cases had poor prognosis. Paired comparative analysis of the case group and the control group revealed that there was a statistical variation in progression between the 2 groups of fetal, which confirmed Nan's view that progressive changes in the width of the lateral ventricle indicated an adverse outcome for the fetus. Devaseelan et al. [21] drew a conclusion from a meta-analysis of 28 clinical studies that fetuses with stable lateral ventricular width had significantly mitigated the risk of neurological abnormalities than fetuses with progressive expansion of lateral ventricular width. However, some people believe that measurement of lateral ventricular width is incapable of prediction of the death of IVM children [22]. This difference is suspected to result from the insufficient sample size, and there remains a lack of uniform standard for the progress of lateral ventricular width, which still requires plenty of prospective analysis and further studies. Progressive IVM needs to take into account the influence of change time. For the same dilatation level, the limited change time of lateral ventricular width indicates that the fetal brain function is possibly more affected than that of the longer change time. In this study, CSWR was one of the contributory factors in the prognosis of fetal VM. This variable was associated with the width difference value of lateral ventricle, the maximum width of lateral ventricle, and the change month, indicating that VM with short-term progress could potentially predict poor prognosis of fetal lateral ventricle dilatation.

Most scholars believe that, for fetuses with IVM, excessively wide or fast growth of lateral ventricles are indicative of poor prognosis of brephyhydrocephalus after birth [21]. Some scholars believe that the size of lateral ventricle width when IVM is first detected and the gestational age when IVM is first diagnosed have predictive

Prognostic and Risk Factors of Fetal Ventriculomegaly values [23]. As discovered by Ouahba et al. [3], atrial width $>12 \mathrm{~mm}$, progression of the enlargement, and asymmetrical and bilateral VM were all 3 factors that could affect the prognosis of children with IVM. In addition to measuring the lateral ventricle width, there are studies using MRI imaging results to predict the prognosis of brephyhydrocephalus [24]. Li et al. [25] conducted a retrospective analysis of 97 cases of fetal IVM, and they claimed that the corpus callosum depth exhibited a negative correlation with the width of lateral ventricle, which could also enable the prediction of the prognosis for children. However, there remains no uniform indicator for the fetal outcome.

Among the fetuses with mild or moderate lateral ventricular enlargement, $1 \sim 14 \%$ of the children with MRI examination can detect the abnormalities with clinical value [2]. Such children need close observation. If the lateral ventricle continues to expand, it will have effect on the prognosis of the fetus, for which most pregnant women choose termination of pregnancy. In this study, 28 out of 164 fetuses had external structure malformation of the forehead (28/164, 17.8\%). Except for 3 fetuses with small arachnoid cysts and 2 fetuses with late gestation, all the other 23 fetuses were termination of pregnancy. The remaining causes of VM include intrauterine infection and genetic or chromosomal abnormalities, which can affect the fetal outcome $[26,27]$. Therefore, in clinical practice, for pregnant women who first find the fetus with VM, clinicians are supposed to recommend relevant prenatal examinations for pregnant women, such as serological examination, amniocentesis for detecting intrauterine infection, and chromosomal microarray analysis to rule out other genetic diseases prior to further treatment.

The birth of newborns will be accompanied by some fetal hydrocephalus into infant hydrocephalus, for which the treatment of infantile hydrocephalus has been a persistent difficulty in neurosurgery. Not only does hydrocephalus cause physical defects in children, it can also make a serious impact on family and society. If prenatal IVM fetus is discovered in pregnant women, timely rehabilitation treatment can alleviate the symptoms of motor function.

\section{Limitations}

A significant limitation of this study is that the followup clinical examinations were performed by the neurosurgeon who conducted the treatment on telephone. A second potential limitation is the exclusion criteria, which included many samples with intraventricular hemorrhage and posthemorrhagic hydrocephalus, it might af- 
fect the result. Another limitation of this study is that intraobserver reliability was not assessed. One final limitation of this study is that it is a retrospective analysis. More samples and longer follow-up prospective studies are required to make further assessment of the prognosis of IVM fetuses.

\section{Conclusions}

It is suggested that the fetal VM should be recommended for regular color Doppler ultrasound examination to measure the relevant indicators of the lateral ventricle. MW and CSWR may be the factor affecting the prognosis of children with hydrocephalus and has the value of predicting fetal prognosis.

\section{Acknowledgement}

We would like to thank Dr. Xue-Wei Xia for his guidance and mentorship.

\section{Statement of Ethics}

The study protocol was approved by the Ethics Committee of Affiliated Hospital of Guilin Medical University and in accordance with the World Medical Association Declaration of Helsinki. The reference number is "2019KS043." Because all the patients includ- ed in this study were children younger than 12 years with brain disorders, their understanding and behavioral ability were limited. So, the informed consent of this study was signed by the parents of the children with the approval of Ethics Committee. We obtained the written consent from the parents of all the participants.

\section{Conflict of Interest Statement}

The authors declare that they have no competing interests.

\section{Funding Sources}

This work was supported by the National Natural Science Foundation of China (No. 81760541 and No. 81860449); the Natural Science Foundation of Guangxi (2017GXNSFBA198001 and 2016GXNSFCA380028); and the China Postdoctoral Science Foundation funded project (2017M613272XB).

\section{Author Contributions}

L.Q.M. prepared the manuscript and follow-up visit. L.Y. designed, interpreted the data, and finally approved the version to be published. X.X.W. assisted to prepare the draft and substantively revised it. L.H.Z. assisted to collect data and performed the statistical analysis. Z.S.Y. assisted in the acquisition of main data. All authors have read and approved the final submitted version. All authors have agreed to be personally accountable for their own contributions and ensure that questions related to the accuracy or integrity of any part of the work shall be answered.

\section{References}

1 Fox NS, Monteagudo A, Kuller JA, Craigo S Norton ME. Mild fetal ventriculomegaly: diagnosis, evaluation, and management. Am J Obstet Gynecol. 2018;219:B2-9.

2 Tully HM, Ishak GE, Rue TC, Dempsey JC Browd SR, Millen KJ, et al. Two hundred thirty-six children with developmental hydrocephalus: causes and clinical consequences. J Child Neurol. 2016;31:309-20.

3 Ouahba J, Luton D, Vuillard E, Garel C, Gressens $\mathrm{P}$, Blanc N, et al. Prenatal isolated mild ventriculomegaly: outcome in 167 cases. BJOG. 2006;113:1072-9.

4 Bar-Yosef O, Barzilay E, Dorembus S, Achiron R, Katorza E. Neurodevelopmental outcome of isolated ventriculomegaly: a prospective cohort study. Prenat Diagn. 2017;37:764-8.

5 Tugcu AU, Gulumser C, Ecevit A, Abbasoglu A, Uysal NS, Kupana ES, et al. Prenatal evaluation and postnatal early outcomes of fetal ventriculomegaly. Eur J Paediatr Neurol. 2014; 18:736-40.

6 Del BM. Ependymal cells: biology and pathology. Acta Neuropathol. 2010;119:55-73.
7 Rodriguez EM, Guerra MM, Vio K, Gonzalez C, Ortloff A, Batiz LF, et al. A cell junction pathology of neural stem cells leads to abnormal neurogenesis and hydrocephalus. Biol Res. 2012;45:231-42.

8 Kousi M, Katsanis N. The genetic basis of hydrocephalus. Annu Rev Neurosci. 2016;39: 409-35.

9 Guerra MM, Henzi R, Ortloff A, Lichtin N, Vio K, Jimenez AJ, et al. Cell junction pathology of neural stem cells is associated with ventricular zone disruption, hydrocephalus, and abnormal neurogenesis. J Neuropathol Exp Neurol. 2015;74:653-71.

10 O'Leary CJ, Nourse CC, Lee NK, White A, Langford M, Sempert K, et al. Neogenin recruitment of the WAVE regulatory complex to ependymal and radial progenitor adherens junctions prevents hydrocephalus. Cell Rep. 2017;20:370-83.

11 Szabolcsi V, Celio MR. De novo expression of parvalbumin in ependymal cells in response to brain injury promotes ependymal remodeling and wound repair. Glia. 2015;63:56794.
12 Del BM. Neuropathology and structural changes in hydrocephalus. Dev Disabil Res Rev. 2010;16:16-22.

13 Farrell TA, Hertzberg BS, Kliewer MA, Harris L, Paine SS. Fetal lateral ventricles: reassessment of normal values for atrial diameter at US. Radiology. 1994;193:409-11.

14 Griffiths PD, Bradburn M, Campbell MJ, Cooper CL, Graham R, Jarvis D, et al. Use of MRI in the diagnosis of fetal brain abnormalities in utero (MERIDIAN): a multicentre, prospective cohort study. Lancet. 2017;389: 538-46.

15 Beeghly M, Ware J, Soul J, du Plessis A, Khwaja O, Senapati GM, et al. Neurodevelopmental outcome of fetuses referred for ventriculomegaly. Ultrasound Obstet Gynecol. 2010;35:405-16.

16 Falip C, Blanc N, Maes E, Zaccaria I, Oury JF, Sebag G, et al. Postnatal clinical and imaging follow-up of infants with prenatal isolated mild ventriculomegaly: a series of 101 cases. Pediatr Radiol. 2007;37:981-9. 
17 Chu N, Zhang Y, Yan Y, Ren Y, Wang L, Zhang B. Fetal ventriculomegaly: pregnancy outcomes and follow-ups in ten years. Biosci Trends. 2016;10:125-32.

18 Carta S, Kaelin AA, Belcaro C, Bhide A. Outcome of fetuses with prenatal diagnosis of isolated severe bilateral ventriculomegaly: systematic review and meta-analysis. Ultrasound Obstet Gynecol. 2018;52:165-73.

19 Melchiorre K, Bhide A, Gika AD, Pilu G, Papageorghiou AT. Counseling in isolated mild fetal ventriculomegaly. Ultrasound Obstet Gynecol. 2009;34:212-24.

20 Sethna F, Tennant PW, Rankin J, C RS. Prevalence, natural history, and clinical outcome of mild to moderate ventriculomegaly. Obstet Gynecol. 2011;117:867-76.
21 Devaseelan P, Cardwell C, Bell B, Ong S. Prognosis of isolated mild to moderate fetal cerebral ventriculomegaly: a systematic review. J Perinat Med. 2010;38:401-9.

22 Hannon T, Tennant PW, Rankin J, Robson SC. Epidemiology, natural history, progression, and postnatal outcome of severe fetal ventriculomegaly. Obstet Gynecol. 2012;120:1345-53.

23 Duru S, Peiro JL, Oria M, Aydin E, Subasi C, Tuncer C, et al. Successful endoscopic third ventriculostomy in children depends on age and etiology of hydrocephalus: outcome analysis in 51 pediatric patients. Childs Nerv Syst. 2018;34:1521-8.

24 Pisapia JM, Akbari H, Rozycki M, Goldstein $\mathrm{H}$, Bakas S, Rathore S, et al. Use of fetal magnetic resonance image analysis and machine learning to predict the need for postnatal cerebrospinal fluid diversion in fetal ventriculomegaly. JAMA Pediatr. 2018;172:128-35.

25 Li H, Liu G, Lin F, Liang H. Formation of the calcarine sulcus: a potential marker to predict the progression in utero of isolated mild fetal ventriculomegaly. Medicine. 2017;96:e7506.

26 Pasquini L, Masini G, Gaini C, Franchi C, Trotta M, Dani C, et al. The utility of infection screening in isolated mild ventriculomegaly: An observational retrospective study on 141 fetuses. Prenat Diagn. 2014;34:1295-300.

27 Donnelly JC, Platt LD, Rebarber A, Zachary J, Grobman WA, Wapner RJ. Association of copy number variants with specific ultrasonographically detected fetal anomalies. Obstet Gynecol. 2014;124:83-90. 